Published in final edited form as:

Diabetes Res Clin Pract. 2018 April ; 138: 177-186. doi:10.1016/j.diabres.2018.02.004.

\title{
Pregnancy Outcomes in Women with an Early Diagnosis of Gestational Diabetes Mellitus
}

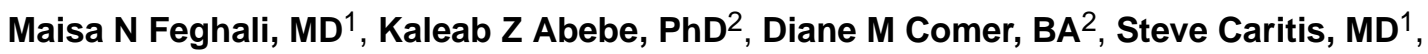 \\ Janet $\mathbf{M}$ Catov, $\mathbf{P h D}^{1}$, and Christina $\mathbf{M}$ Scifres, $\mathbf{M D}^{1,3}$ \\ ${ }^{1}$ Department of Obstetrics, Gynecology and Reproductive Sciences, Magee-Womens Research \\ Institute, University of Pittsburgh School of Medicine \\ ${ }^{2}$ Department of Medicine, University of Pittsburgh School of Medicine \\ ${ }^{3}$ Department of Obstetrics and Gynecology, University of Oklahoma College of Medicine
}

\section{Abstract}

Aim-To examine pregnancy outcomes in women with gestational diabetes mellitus (GDM) based on the timing of diagnosis.

Method-We compared demographics, blood sugars and outcomes between women diagnosed before ( $\mathrm{n}=167)$ or after 24 weeks' gestation ( $\mathrm{n}=1202)$ in a single hospital between 2009 and 2012. Because early screening is risk-based we used propensity score modelling and conditional logistic regression to account for systematic differences.

\begin{abstract}
Results-Women diagnosed with GDM before 24 weeks were more likely to be obese and they were less likely to have excess gestational weight gain ( $35 \mathrm{vs.} 45 \%$, p=0.04). Early diagnosis was associated with more frequent therapy including glyburide (65 vs. $56 \%$, p $<0.001)$ and insulin $(19$ vs $6 \%, \mathrm{p}<0.001)$. After propensity score modelling and accounting for covariates, early diagnosis was associated with an increased risk for macrosomia (OR 2, 95\% 1-4.15, p=0.0498). Early diagnosis was not associated with other adverse outcomes. In a subgroup analysis comparing women treated with glyburide prior to 24 weeks compared to those diagnosed after 24 weeks, early diagnosis in women treated with glyburide was associated with an increased risk for macrosomia (OR 2.3, 95\% CI 1.1-5.4, P=0.04).
\end{abstract}

Conclusion-Women diagnosed with GDM before 24 weeks have unique features, are at risk for adverse outcomes, and require targeted approaches to therapy.

\section{Keywords}

Gestational Diabetes; Diagnosis; Pregnancy

Corresponding author: Maisa Feghali, MD, 300 Halket St, Pittsburgh, PA 15213, maisafeghali@ gmail.com, Phone: 4126414874 , Fax: 4126411504.

Publisher's Disclaimer: This is a PDF file of an unedited manuscript that has been accepted for publication. As a service to our customers we are providing this early version of the manuscript. The manuscript will undergo copyediting, typesetting, and review of the resulting proof before it is published in its final citable form. Please note that during the production process errors may be discovered which could affect the content, and all legal disclaimers that apply to the journal pertain.

The authors report no conflict of interest.

This work was presented in part at the $75^{\text {th }}$ Annual American Diabetes Association Scientific Meeting, June 2015, Boston, MA. 


\section{Introduction}

Treatment of gestational diabetes mellitus (GDM) improves maternal and neonatal outcomes. ${ }^{1-3}$ While there is an ongoing debate regarding the pptimal GDM screening strategy, ${ }^{4}$ numerous professional societies now support universal GDM screening between 24-28 weeks of gestation. ${ }^{5-8}$ Insulin resistance increases with advancing gestation, ${ }^{9}$ and screening at 24-28 weeks is recommended to coincide with peak insulin resistance while allowing sufficient time for treatment benefit. However, the higher prevalence of obesity and diabetes outside of pregnancy raises concern that some pregnant women may develop gestational diabetes prior to 24 weeks' gestation or present with undiagnosed pre-gestational diabetes. Several professional organizations recommend diabetes testing at the first prenatal visit for either all women ${ }^{8}$ or those with risk factors such as age greater than 35 , obesity, prior GDM, previous macrosomic infant, family history of diabetes and PCOS. ${ }^{5,7}$ Higher first trimester fasting glucose levels, even below those typically diagnostic of diabetes, increase the risk for LGA birth weight, macrosomia, and cesarean delivery ${ }^{10}$. This knowledge has prompted many physicians to treat women diagnosed with GDM prior to 24 weeks, and these women may have more advanced pathophysiology and a higher risk for poor maternal and neonatal outcomes.

In a recent study, Sweeting et. al. described higher rates of adverse pregnancy outcomes in women diagnosed with GDM before 24 weeks, with the highest risk occurring in those women diagnosed with GDM at less than 12 weeks. ${ }^{11}$ However, after accounting for baseline maternal characteristics including maternal obesity, gestational weight gain, and fasting glucose the timing of GDM diagnosis was no longer associated with differences in large for gestational age (LGA) birth weight or macrosomia. ${ }^{11}$ Women in this cohort were exclusively treated with insulin, which is in contrast to the United States where glyburide has become the most common treatment for GDM in recent years. ${ }^{12}$ Therefore, we set out to better characterize treatment patterns and maternal and neonatal outcomes between women with an earlier ( $<24$ weeks) and later ( $\geq 24$ weeks) diagnosis of gestational diabetes in a US cohort.

\section{Material and methods}

This was a secondary analysis of retrospective cohort study created to examine the clinical course and outcomes of a contemporary, well-characterized population of patients with GDM. Women with singleton gestations and GDM who were delivered at Magee-Womens Hospital (University of Pittsburgh, Pittsburgh, PA) from January 2009 to October 2012 were included. As previously described, women were identified using the ICD-9 codes 648.01 (diabetes-delivered) and 648.81 (abnormal glucose tolerance-delivered), and medical records were reviewed to confirm the diabetes diagnosi. ${ }^{13}$ Women were deemed to have pregestational diabetes if they reported a diagnosis of diabetes at their first prenatal visit or if they had a first trimester $\mathrm{HbA} 1 \mathrm{c}$ value $\varangle 6.5 \%(48 \mathrm{mmol} / \mathrm{mol})$, and there were a total of 20 women excluded for this reason. Women with pre-gestational diabetes were excluded, and those with GDM were included only if their records were available for review and if they had either a 50-g one-hour glucose challenge test (GCT) that exceeded $200 \mathrm{mg} / \mathrm{dL}$, or if they 
had two or more abnormal values on a 3 hour, 100 gram oral glucose tolerance test (OGTT) as defined by the Carpenter-Coustan Criteria. ${ }^{5}$ Out of a total of 38,222 deliveries, we identified 1374 women with GDM, and only the first pregnancy during the study period was included. There were 5 women who were excluded because the precise timing of their GDM testing was unknown, leaving 1,369 women for the final cohort. Regulatory approval was obtained from the University of Pittsburgh Institutional Review Board, and informed consent was not required given the retrospective nature of the study.

Women included in this study received prenatal care in the obstetric and maternal fetal medicine clinics at our hospital. Early GDM screening was performed at the discretion of the provider, and the majority of women underwent GDM testing using a non-fasting, $50 \mathrm{~g}$ glucose challenge test (GCT) followed by a fasting, $100 \mathrm{~g}$ oral glucose tolerance test (OGTT). GDM diagnosis was established by either a GCT that exceeded $200 \mathrm{mg} / \mathrm{dL}$ as per institutional policy, or if they had two or more abnormal values on a 3 hour, 100 gram OGTT as defined by the Carpenter-Coustan Criteria. ${ }^{14}$ The majority of women with GDM ( $\mathrm{n}=1215$, $88.7 \%$ ) received their nutritional counseling through a centralized office where they were given instructions regarding their diet and recommended weight gain based on their prepregnancy BMI. The remainder received similar counseling but in separate locations. Selfmonitoring of plasma glucose was recommended four times daily, and targets for plasma glucose included a fasting value less than $95 \mathrm{mg} / \mathrm{dL}$ and one-hour post-meal values less than $140 \mathrm{mg} / \mathrm{dL} .^{5}$

Pre-pregnancy BMI was calculated from the pre-pregnancy weight reported in the medical record, and the reported pre-pregnancy weight had a strong correlation with the measured weight at the first prenatal visit $(r=0.98, p<0.001)$. Maternal pre-pregnancy overweight and obesity was reported as an index of weight-for-height (body mass index, BMI), and overweight/obesity was defined using the WHO guidelines for classification of BMI. ${ }^{15}$ Gestational weight gain was defined as insufficient, sufficient, or excessive for each prepregnancy BMI category as defined in the Institute of Medicine 2009 guidelines. ${ }^{16}$ In order to assess the association between excess gestational weight gain and pregnancy outcomes in those women who delivered preterm we estimated the maximal recommended weight gain at the gestational age at which they were delivered. We performed these calculations by multiplying the maximal weekly weight gain in the second and third trimesters times the number of weeks preterm the patient was delivered and subtracting this value from the maximum recommended weight gain for each BMI category. ${ }^{17}$ To assess maternal glycemic control, 7 days of consecutive blood sugars were obtained from the medical record at 4 week intervals. Blood sugar data were available for $1147 / 1369$ women $(83.8 \%)$, and the mean fasting and postprandial blood sugars were calculated across gestation. We also obtained information regarding medication use including dose and gestational age at initiation of therapy and type and dose of medication at delivery.

Our primary pregnancy outcomes included macrosomia, preterm delivery, hypertensive disorders of pregnancy, and neonatal morbidity. Macrosomia was defined as birth weight $>4000$ grams, and we also compared large for gestational age $\left(>90^{\text {th }}\right.$ percentile for gestational age) or small for gestational age $\left(<10^{\text {th }}\right.$ percentile for gestational age $)$ birth weight status based on US national birth weight data between those women with an early 
GDM diagnosis and those who were diagnosed after 24 weeks. ${ }^{18}$ Preterm births ( $<37$ weeks) were further characterized as spontaneous (following the spontaneous onset of contractions or premature rupture of membranes) or indicated preterm birth, which encompassed all other preterm deliveries. Hypertensive disorders of pregnancy were considered together as a single outcome consisting of new onset blood pressures $\geq 140 / 90 \mathrm{mmHg}$ on two or more occasions six hours apart after 20 weeks' gestation with or without proteinuria or blood pressure exacerbations along with new-onset proteinuria ( $\searrow 0.3 \mathrm{~g} / 24$ hours) in women with chronic hypertension. Neonatal outcomes included neonatal intensive care unit (NICU) admission, hypoglycemia (defined as a glucose value less than $35 \mathrm{mg} / \mathrm{dL}$ within the first 24 hours of life), hyperbilirubinemia requiring phototherapy, need for supplemental oxygen or other respiratory support beyond 24 hours of life, congenital anomalies, and neonatal death. We also defined a composite neonatal morbidity consisting of hypoglycemia, hyperbilirubinemia, or respiratory morbidity. Other outcomes considered included primary cesarean delivery, stillbirth, and shoulder dystocia.

Statistical analyses were completed using Stata 13 software package Special Edition (StataCorp LP, College Station, TX) and SAS Version 9.4 (SAS Institute Inc, Cary, NC). Distributions of variables were tested for normality using visual inspection of histograms and the Shapiro-Wilk $W$-test. We first categorized women into two groups based on the timing of their GDM diagnosis (< vs $\geq 24$ weeks). Baseline characteristics and demographics of women were compared by group using chi-squared statistics, two-sample t-tests, or their nonparametric equivalents.

In order to account for imbalances between groups on baseline characteristics, we utilized a propensity score model with inverse probability of treatment weighting to create a subcohort of women who were well-balanced on all measured covariates. ${ }^{19,20}$ Propensity scores were calculated for each woman using logistic regression. This modeled the probability of GDM diagnosis being < 24 weeks as a function of: maternal age, maternal race, education ( $\geq$ some college), private insurance, nulliparity, pre-pregnancy BMI, tobacco use, presence of chronic hypertension, prior history of GDM, and 50gm GCT value. Each estimated propensity score was weighted by the inverse probability of being diagnosed with GDM before or after 24 weeks. In order to assess for balance, we calculated weighted standardized mean differences for each of the baseline covariates and compared the magnitude of imbalance to the unweighted differences.

Conditional logistic regression was used to assess the relationship between timing of diagnosis (before vs after 24 weeks) and each of the dichotomous perinatal outcomes (macrosomia, preterm birth, hypertensive disorders of pregnancy, and neonatal morbidity). The impact of timing on the birthweight z-score was assessed using a linear mixed model with a random effect for the matched pair. Both models included single fixed effect for timing of diagnosis (before vs after 24 weeks). Because there is a paucity of data regarding glyburide use early in pregnancy when compared to later use, we also conducted a sensitivity analysis comparing rates of pregnancy outcomes between timing groups among the 701 women treated with glyburide. Two-sided p-values less than 0.05 were considered statistically significant in all analyses. 


\section{Results}

We included 1,369 women with GDM in our primary analyses, and of these women 167 (12.3\%) were diagnosed prior to 24 weeks. Prior to propensity score modelling, women who had an early diagnosis were older, less likely to have any college education or private insurance, and less likely to be nulliparous. Women with an early diagnosis of GDM were more likely to be obese, and they were less likely to have excess gestational weight gain (Table 1). Women with an earlier diagnosis of GDM were also more likely to have chronic hypertension, and among women with a prior pregnancy and information regarding a history of GDM ( $n=680$ ), women with an early diagnosis were more likely to have been diagnosed with GDM in a prior pregnancy. Women with an early diagnosis of GDM had higher glucose values on their 50-g glucose challenge test as well as higher values on their fasting and 1hour glucose values on their oral glucose tolerance test. Table 1 also highlights how baseline differences in maternal pre-pregnancy BMI and history of GDM can be attenuated and therefore less likely to influence associations with outcome after propensity score modeling.

HbA1c testing was not standard in women with GDM at our institution, but 120/1369 $(8.8 \%)$ of women had at least one HbA1c value checked during pregnancy and the twenty women with a $\mathrm{HbA} 1 \mathrm{c}$ value $\ 6.5 \%$ were excluded. Women diagnosed before 24 weeks were more likely to have had an $\mathrm{HbA} 1 \mathrm{c}$ measured than those with diagnosis after 24 weeks $(56 / 167(33.5 \%)$ vs $64 / 1202(5.3 \%), \mathrm{p}<0.001)$. HbA1c values were measured earlier in those diagnosed <24 weeks ( $16.1 \pm 7.1$ vs $29.7 \pm 5.9$ weeks, $\mathrm{p}<0.001)$, and $\mathrm{HbA1c}$ values were similar between women with a GDM diagnosis before 24 weeks compared with after 24 weeks $(6.1 \pm 0.9 \%(43 \mathrm{mmol} / \mathrm{mol})$ vs $5.9 \pm 0.7 \%$ ( $41 \mathrm{mmol} / \mathrm{mol}), \mathrm{p}=0.21)$. There were also significant differences in the type of therapy women were using at delivery, with fewer diagnosed before 24 weeks managed with dietary therapy and more women requiring either glyburide or insulin (Table 1). Women who were diagnosed with GDM before 24 weeks had higher mean fasting and post-prandial blood sugars across gestation. As expected, betweengroup differences including differences in glycemic control after diagnosis, except for need for pharmacologic therapy, vanished after propensity score modelling (Table 1).

Prior to propensity score modelling women who were diagnosed before 24 weeks were at increased risk for preterm birth, LGA birth weight, macrosomia, hypertensive disorders of pregnancy, NICU admission, and neonatal composite morbidity (Table 2). After propensity score modelling, there were few differences in pregnancy outcomes between women diagnosed at less than 24 weeks or after 24 weeks (Table 2). Gestational age at delivery was slightly lower ( $37.7 \pm 0.7$ vs. $38.4 \pm 0.1$ weeks, $\mathrm{p}=0.008)$ in women diagnosed with GDM prior to 24 weeks (Table 2). Macrosomia was more common in the early diagnosis group $(14.8 \%$ vs $7.8 \%, \mathrm{p}=0.049)$, and this difference persisted after logistic regression analysis (OR 2, 95\% 1-4.15, $\mathrm{p}=0.05$ ) (Table 3).

Because there is a paucity of data regarding glyburide use before 24 weeks, we conducted a subgroup analysis exploring outcomes in this group. Women diagnosed with GDM prior to 24 weeks and treated with glyburide were compared to those diagnosed with GDM after 24 weeks and treated with glyburide (Table 4). Prior to propensity score modelling, women treated with glyburide before 24 weeks had higher rates of macrosomia, preterm birth, and 
neonatal morbidity compared to women prescribed glyburide in the standard diagnosis group (Table 5). After propensity score modelling, early diagnosis of GDM in women treated with glyburide was associated with an increased risk for macrosomia (OR 2.3, 95\% CI 1.1-5.4, $\mathrm{P}=0.04)$. However, timing of diagnosis was not associated with a higher risk for other adverse pregnancy outcomes including hypertensive disorders pregnancy, preterm birth, or neonatal morbidity (Table 6).

Because of the possibility that very early diagnosis ( $<13$ weeks) may be associated with worse outcomes compared to those diagnosed between 13-24 weeks, we also performed an analysis comparing the prevalence of selected outcomes between those women diagnosed $<13$ weeks, 13-23.9 weeks, and greater than or equal to 24 weeks. Of women diagnosed before 24 weeks, 52/167 (31.3\%) were diagnosed at $<13$ weeks. Women who were diagnosed after 24 weeks were at lower risk for adverse outcomes such as rates of preterm birth ( 12.8 vs 21.6 vs $25.4 \%$, p<0.001), macrosomia ( 7.0 vs 11.5 vs $13.0 \%, p=0.04)$, NICU admission ( 10.8 vs 18.0 vs $21.4 \%, \mathrm{p}=0.002$ ), and composite neonatal morbidity ( 18.3 vs 27.5 vs $25.2 \%, \mathrm{p}=0.06$ ) when compared to women who were diagnosed at $<13$ weeks or between 13 and 23.9 weeks.

\section{Discussion}

We found that women who were diagnosed with GDM before 24 weeks were at higher risk of macrosomia after accounting for baseline differences using propensity weighting. However, timing of diagnosis was not associated with an increased risk for other adverse outcomes including hypertensive disorders of pregnancy, preterm birth, or neonatal morbidity. Various factors may underlie the increased risk for macrosomia in women with an early diagnosis of GDM. In addition to glycemic control, pre-pregnancy obesity and excess gestational weight gain are associated with increased risk for macrosomia. ${ }^{21,} 22$ In our study, the increase risk for macrosomia persisted after we accounted for differences in prepregnancy BMI. Women with an early diagnosis of GDM had higher blood glucose values across gestation, but these differences were attenuated after our propensity score modeling. Early diagnosis of GDM was associated with lower rates of excess weight gain and higher rates of inadequate weight gain, suggesting that nutritional counseling earlier in gestation impacts maternal behavior. However, these differences in weight gain were insufficient to reduce the risk for macrosomia.

Despite lower weight gain, women with an early diagnosis of GDM were more likely to require medical therapy, and glyburide was the most common agent utilized in women with GDM regardless of timing of diagnosis. Approximately a quarter of women who were started on glyburide prior to 24 weeks required insulin before delivery, whereas very few women who started on glyburide after 24 weeks required a change to insulin therapy. These findings are consistent with prior studies that suggested a higher rate of glyburide failure in women diagnosed before 25 weeks of gestation. ${ }^{23-26}$ While early data suggested similar outcomes among women treated with either glyburide or insulin, ${ }^{24}$ recent reports described a 2 -fold increased risk for macrosomia with glyburide compared to insulin therapy. ${ }^{27,} 28$ Importantly, little data is available on the use of glyburide in women during early pregnancy. Glycemic control was similar between women treated with glyburide in the early and 
standard diagnosis groups, and this raises the concern that transplacental glyburide and fetal exposure could contribute to this overgrowth. ${ }^{29-31}$ Women in the early diagnosis group received glyburide at different stages in pregnancy, at higher doses and for longer periods of time. It is possible that other metabolic factors or unmeasured hyperglycemia contributed to the risk for macrosomia in women with an early diagnosis, but further data are required to assess the risks and benefits of glyburide use in early gestation.

Current strategies for GDM screening are based on the gradual increase in insulin resistance and manifestation of hyperglycemia that occurs as pregnancy progresses, ${ }^{9}$ and women who undergo early screening are more likely to have multiple risk factors for adverse outcomes. Early pregnancy is a critical window in development, as evidenced by recent data demonstrating that maternal insulin response in early pregnancy is associated with early pregnancy placental volume and placental weight at birth. ${ }^{32}$ It is therefore possible that some of the adverse outcomes in women with early diagnosis of GDM relate to programming events in early pregnancy that may be more resistant to intervention once GDM is diagnosed. In addition, the optimal strategy for diabetes diagnosis in early pregnancy is unknown. The IADPSG has recommended glycemic cut-offs for diagnosing gestational diabetes in early pregnancy, but more recent reports have challenged this recommendation due to the observation that early fasting plasma glucose was poorly predictive of glycemic status beyond 24 weeks. ${ }^{33-35}$ Many major organization advocate treating only those women with "overt" or evidence of pre-gestational diabetes, but our findings demonstrate that there is a population of women with GDM earlier in pregnancy who are at higher risk for adverse outcomes. ${ }^{5,7,8}$

Because early screening for GDM is risk-based, we utilized inverse probability of treatment weighting to account for the baseline imbalances in maternal characteristics between women diagnosed with GDM before and after 24 weeks. This strategy has been successfully employed in other disciplines such as the cardiovascular literature ${ }^{36}$ to address some of the baseline imbalances that occur in observational studies. However, one limitation to inverse probability of treatment weighting is that is cannot account for unmeasured confounding, and although we were able to account for a broad number of clinical and demographic variables it is possible that there are metabolic factors that we were unable to account for in women diagnosed with GDM before 24 weeks. Other limitations are that HbA1c screening was not universally performed, and it is possible that we missed some cases of overt diabetes. ${ }^{7}$ Our data suggests that women who underwent early diagnosis at $<13$ weeks were at similar risk for adverse outcomes as those diagnosed between 13 and 23.9 weeks; gestation, although these analyses were limited by the small number of women diagnosed at less than 13 weeks. Also, there were a small number of women treated with insulin in either the early or standard diagnosis groups, which limited our ability to compare outcomes between treatment strategies or to examine outcomes in women diagnosed before and after 24 weeks treated with insulin. We also did not have information on post-partum testing, which limited our ability to compare the risk for ongoing type 2 diabetes among groups. Conversely, significant strengths of our study are the overall cohort size, the use of a propensity score model, the matched subcohort analysis, and the inclusion of glycemic control and data on pharmacologic treatment throughout gestation. 
Our findings are important because the prevalence of GDM is increasing and counselling regarding risk and decisions regarding treatment have both maternal and neonatal effects. Using a risk-based strategy for early screening, women diagnosed with GDM prior to 24 weeks are a group at particular risk for adverse outcomes, and they require targeted approaches to therapy. We suggest caution with use of glyburide in women with an early diagnosis of GDM until further studies regarding glyburide use prior to 24 weeks are available. There is a paucity of data regarding optimal glycemic targets for high-risk women with diabetes in pregnancy, and it is possible that different glycemic targets or alternate therapeutic approaches are needed in this population. ${ }^{37}$ Further studies are also needed to establish the risks and benefits of early diabetes screening and treatment.

\section{Acknowledgments}

M.N.F. is supported by the National Institutes of Health through Grant Number KL2 TR001856. The funding source had no involvement in the preparation, analysis, and interpretation of the data or submission of this report.

\section{References}

1. Crowther CA, Hiller JE, Moss JR, et al. Effect of treatment of gestational diabetes mellitus on pregnancy outcomes. N Engl J Med. 2005; 352:2477-86. [PubMed: 15951574]

2. Landon MB, Spong CY, Thom E, et al. A multicenter, randomized trial of treatment for mild gestational diabetes. N Engl J Med. 2009; 361:1339-48. [PubMed: 19797280]

3. Tieu J, McPhee AJ, Crowther CA, Middleton P. Screening and subsequent management for gestational diabetes for improving maternal and infant health. Cochrane Database Syst Rev. 2014:CD007222. [PubMed: 24515533]

4. Saccone G, Caissutti C, Khalifeh A, et al. One step versus two step approach for gestational diabetes screening: systematic review and meta-analysis of the randomized trials. J Matern Fetal Neonatal Med. 2017:1-9.

5. Committee on Practice B-O. Practice Bulletin No. 137: Gestational diabetes mellitus. Obstet Gynecol. 2013; 122:406-16. [PubMed: 23969827]

6. Moyer VA, Force USPST. Screening for gestational diabetes mellitus: U.S. Preventive Services Task Force recommendation statement. Annals of internal medicine. 2014; 160:414-20. [PubMed: 24424622]

7. Standards of Medical Care in Diabetes-2017: Summary of Revisions. Diabetes Care. 2017; 40:S4s5. [PubMed: 27979887]

8. Blumer I, Hadar E, Hadden DR, et al. Diabetes and pregnancy: an endocrine society clinical practice guideline. J Clin Endocrinol Metab. 2013; 98:4227-49. [PubMed: 24194617]

9. Catalano PM, Tyzbir ED, Wolfe RR, et al. Carbohydrate metabolism during pregnancy in control subjects and women with gestational diabetes. Am J Physiol. 1993; 264:E60-7. [PubMed: 8430789]

10. Riskin-Mashiah S, Younes G, Damti A, Auslender R. First-trimester fasting hyperglycemia and adverse pregnancy outcomes. Diabetes Care. 2009; 32:1639-43. [PubMed: 19549728]

11. Sweeting AN, Ross GP, Hyett J, et al. Gestational Diabetes Mellitus in Early Pregnancy: Evidence for Poor Pregnancy Outcomes Despite Treatment. Diabetes care. 2016; 39:75-81. [PubMed: 26645084]

12. Camelo Castillo W, Boggess K, Sturmer T, Brookhart MA, Benjamin DK Jr, Jonsson Funk M. Trends in glyburide compared with insulin use for gestational diabetes treatment in the United States, 2000-2011. Obstet Gynecol. 2014; 123:1177-84. [PubMed: 24807336]

13. Scifres C, Feghali M, Althouse AD, Caritis S, Catov J. Adverse Outcomes and Potential Targets for Intervention in Gestational Diabetes and Obesity. Obstet Gynecol. 2015; 126:316-25. [PubMed: 26241421]

14. Carpenter MW, Coustan DR. Criteria for screening tests for gestational diabetes. American journal of obstetrics and gynecology. 1982; 144:768-73. [PubMed: 7148898] 
15. Physical status: the use and interpretation of anthropometry. Report of a WHO Expert Committee. 1995; 854:1-452. World Health Organization technical report series

16. Rasmussen KM, Catalano PM, Yaktine AL. New guidelines for weight gain during pregnancy: what obstetrician/gynecologists should know. Curr Opin Obstet Gynecol. 2009; 21:521-6. [PubMed: 19809317]

17. Scifres CM, Feghali MN, Althouse AD, Caritis SN, Catov JM. Effect of excess gestational weight gain on pregnancy outcomes in women with type 1 diabetes. Obstet Gynecol. 2014; 123:1295302. [PubMed: 24807331]

18. Alexander GR, Himes JH, Kaufman RB, Mor J, Kogan M. A United States national reference for fetal growth. Obstet Gynecol. 1996; 87:163-8. [PubMed: 8559516]

19. Austin PC, Stuart EA. Moving towards best practice when using inverse probability of treatment weighting (IPTW) using the propensity score to estimate causal treatment effects in observational studies. Statistics in medicine. 2015; 34:3661-79. [PubMed: 26238958]

20. Rosenbaum PR, R DB. The central role of propensity score in observational studies for causal effects. Biometrika. 1983; 70:41-55.

21. Hyperglycaemia and Adverse Pregnancy Outcome (HAPO) Study: associations with maternal body mass index. Bjog. 2010; 117:575-84. [PubMed: 20089115]

22. Badon SE, Dyer AR, Josefson JL. Gestational weight gain and neonatal adiposity in the Hyperglycemia and Adverse Pregnancy Outcome study-North American region. Obesity (Silver Spring). 2014; 22:1731-8. [PubMed: 24634400]

23. Kahn BF, Davies JK, Lynch AM, Reynolds RM, Barbour LA. Predictors of glyburide failure in the treatment of gestational diabetes. Obstet Gynecol. 2006; 107:1303-9. [PubMed: 16738156]

24. Langer O, Conway DL, Berkus MD, Xenakis EM, Gonzales O. A comparison of glyburide and insulin in women with gestational diabetes mellitus. N Engl J Med. 2000; 343:1134-8. [PubMed: 11036118]

25. Chmait R, Dinise T, Moore T. Prospective observational study to establish predictors of glyburide success in women with gestational diabetes mellitus. J Perinatol. 2004; 24:617-22. [PubMed: 15152273]

26. Rochon M, Rand L, Roth L, Gaddipati S. Glyburide for the management of gestational diabetes: risk factors predictive of failure and associated pregnancy outcomes. Am J Obstet Gynecol. 2006; 195:1090-4. [PubMed: 17000241]

27. Balsells M, Garcia-Patterson A, Sola I, Roque M, Gich I, Corcoy R. Glibenclamide, metformin, and insulin for the treatment of gestational diabetes: a systematic review and meta-analysis. BMJ (Clinical research ed). 2015; 350:h102.

28. Camelo Castillo W, Boggess K, Sturmer T, Brookhart MA, Benjamin DK Jr, Jonsson Funk M. Association of Adverse Pregnancy Outcomes With Glyburide vs Insulin in Women With Gestational Diabetes. JAMA Pediatr. 2015; 169:452-8. [PubMed: 25822253]

29. Caritis SN, Hebert MF. A pharmacologic approach to the use of glyburide in pregnancy. Obstetrics and gynecology. 2013; 121:1309-12. [PubMed: 23812467]

30. Schwartz RA, Rosenn B, Aleksa K, Koren G. Glyburide transport across the human placenta. Obstetrics and gynecology. 2015; 125:583-8. [PubMed: 25730219]

31. Hebert MF, Ma X, Naraharisetti SB, et al. Are we optimizing gestational diabetes treatment with glyburide? The pharmacologic basis for better clinical practice. Clinical pharmacology and therapeutics. 2009; 85:607-14. [PubMed: 19295505]

32. O'Tierney-Ginn P, Presley L, Myers S, Catalano P. Placental growth response to maternal insulin in early pregnancy. The Journal of clinical endocrinology and metabolism. 2015; 100:159-65. [PubMed: 25365315]

33. Corrado F, D’Anna R, Cannata ML, Interdonato ML, Pintaudi B, Di Benedetto A. Correspondence between first-trimester fasting glycaemia, and oral glucose tolerance test in gestational diabetes diagnosis. Diabetes Metab. 2012; 38:458-61. [PubMed: 22595470]

34. International Association of D; Pregnancy Study Groups Consensus P. Metzger BE, et al. International association of diabetes and pregnancy study groups recommendations on the diagnosis and classification of hyperglycemia in pregnancy. Diabetes Care. 2010; 33:676-82. [PubMed: 20190296] 
35. Zhu WW, Yang HX, Wei YM, et al. Evaluation of the value of fasting plasma glucose in the first prenatal visit to diagnose gestational diabetes mellitus in china. Diabetes Care. 2013; 36:586-90. [PubMed: 23193214]

36. Bangalore S, Bhatt DL, Rother J, et al. Late outcomes after carotid artery stenting versus carotid endarterectomy: insights from a propensity-matched analysis of the Reduction of Atherothrombosis for Continued Health (REACH) Registry. Circulation. 2010; 122:1091-100. [PubMed: 20805431]

37. Martis R, Brown J, Alsweiler J, Crawford TJ, Crowther CA. Different intensities of glycaemic control for women with gestational diabetes mellitus. Cochrane Database Syst Rev. 2016; 4:CD011624. [PubMed: 27055233] 


\section{Highlights}

Women diagnosed with gestational diabetes mellitus before 24 weeks are at increased risk for adverse outcomes, specifically macrosomia, and they require targeted approaches to therapy. 


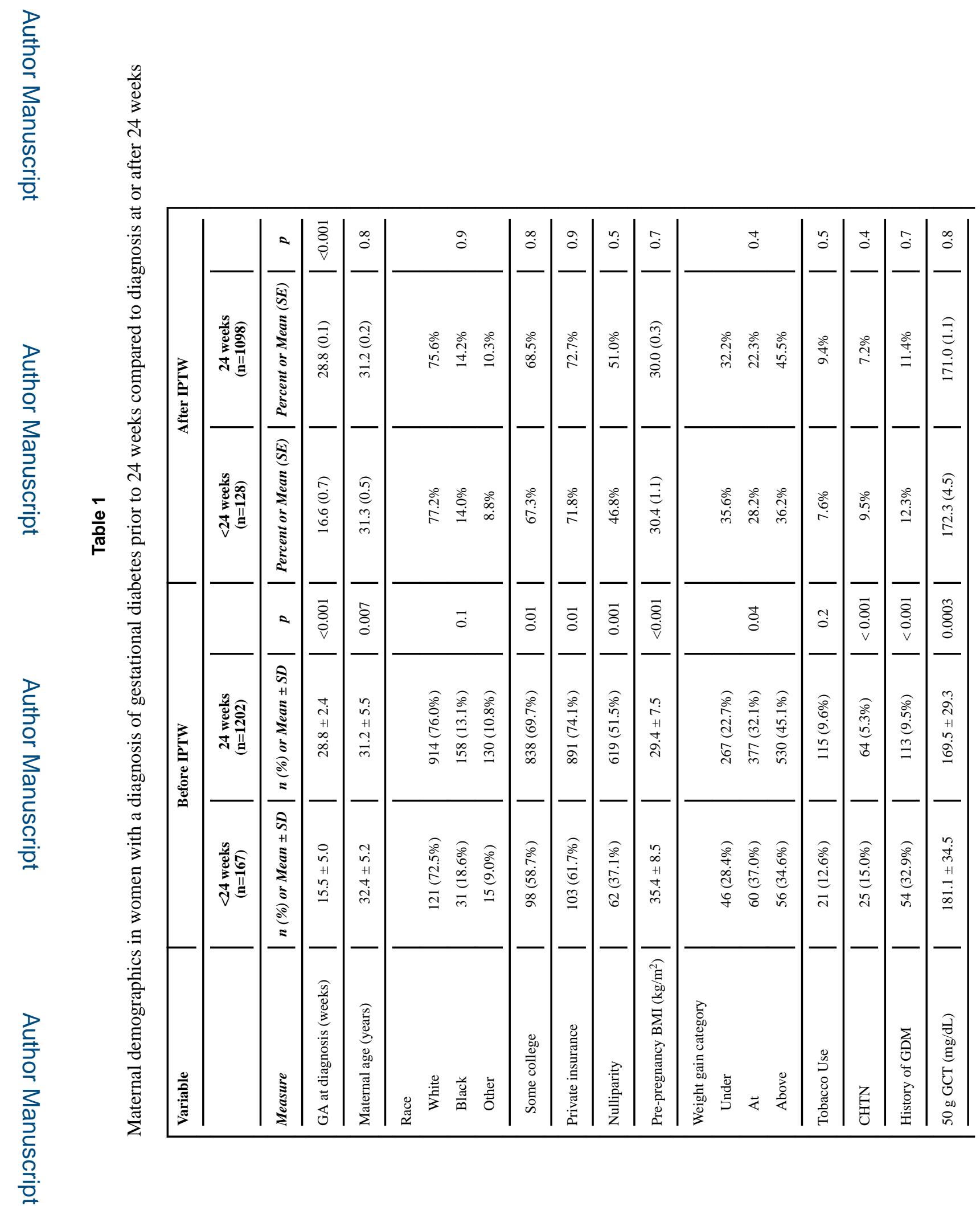

Diabetes Res Clin Pract. Author manuscript; available in PMC 2019 April 01. 
을

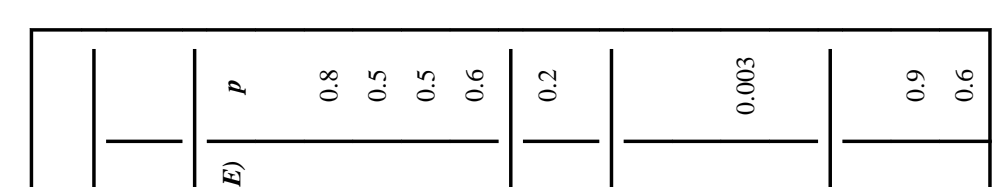

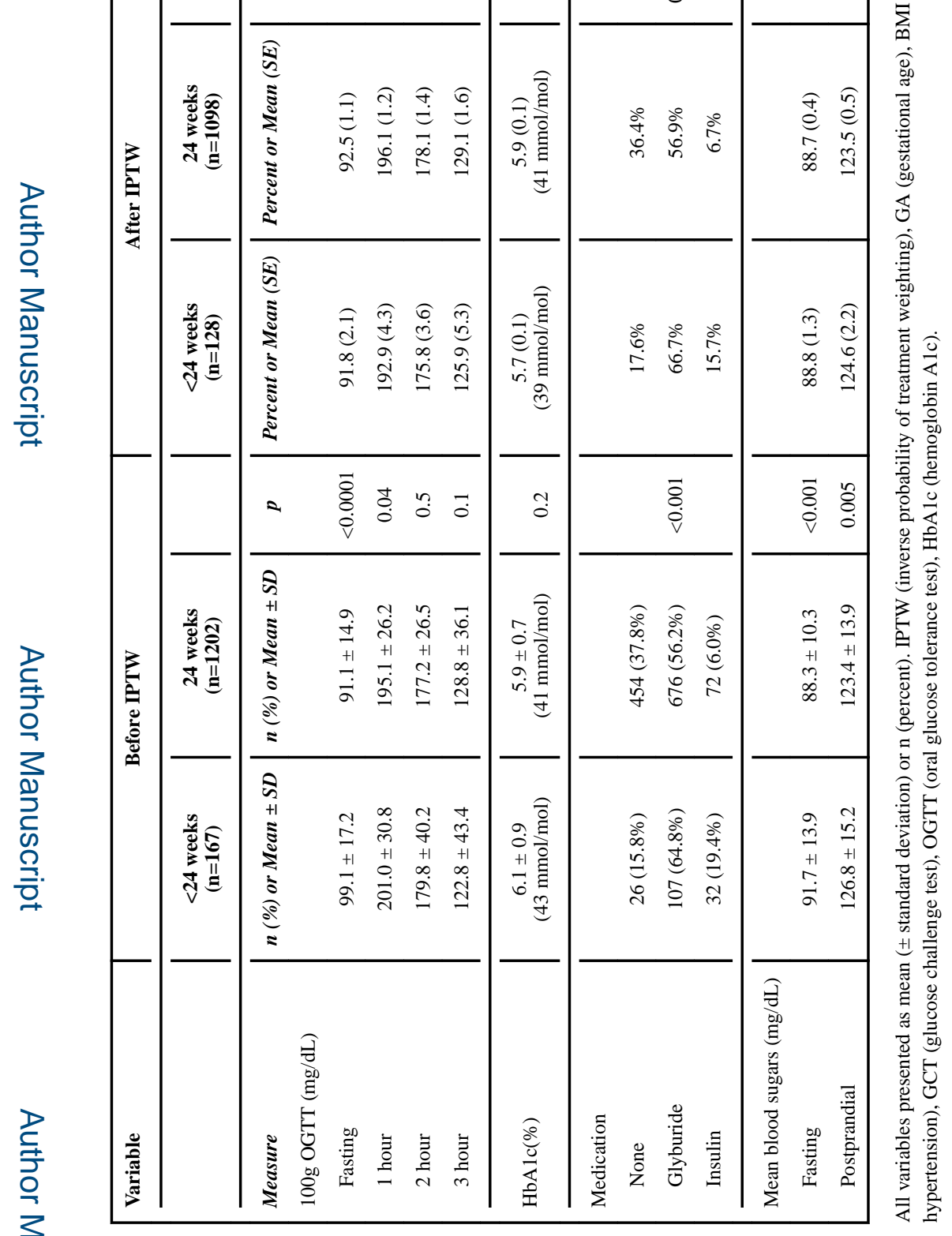




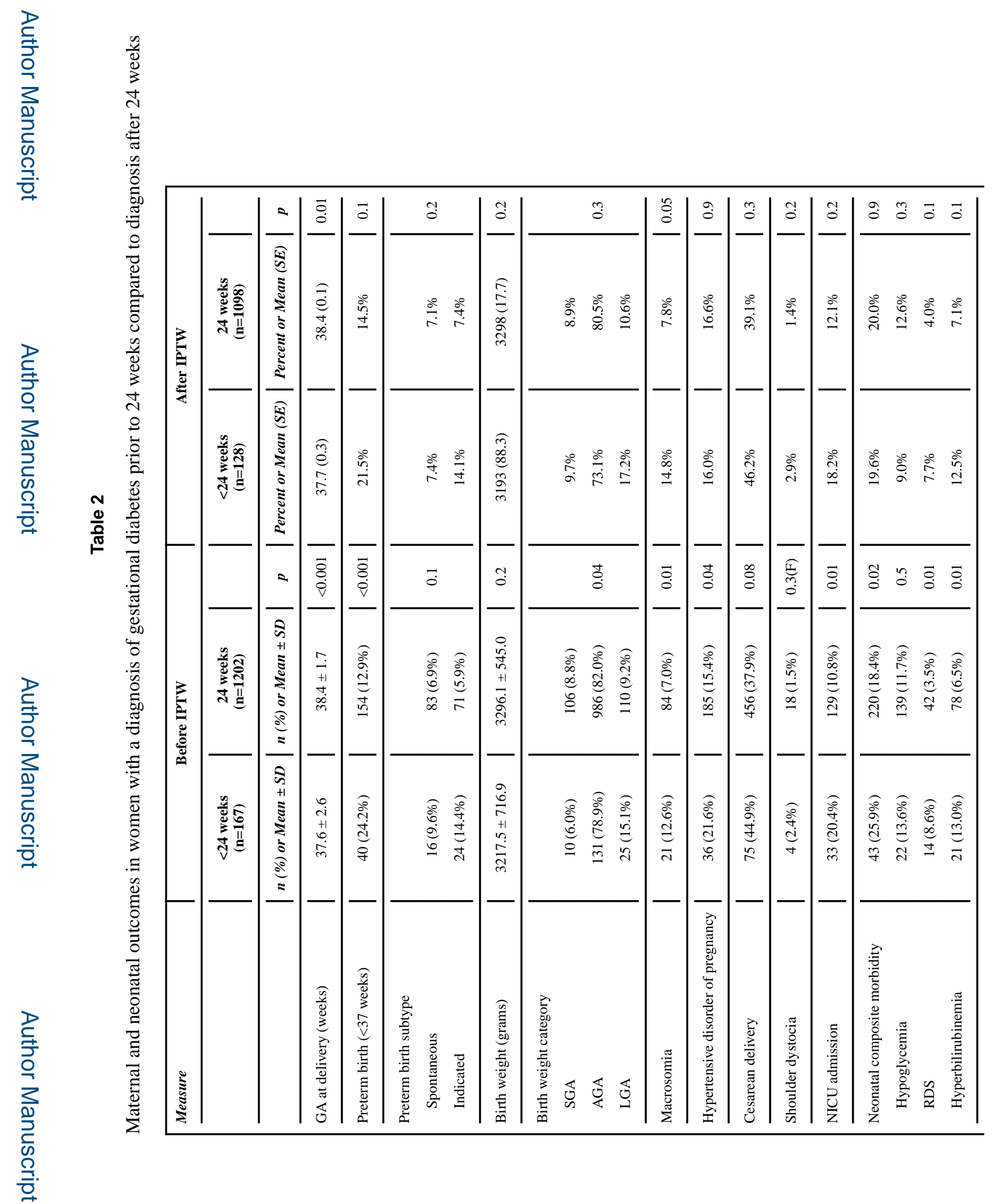

Diabetes Res Clin Pract. Author manuscript; available in PMC 2019 April 01. 


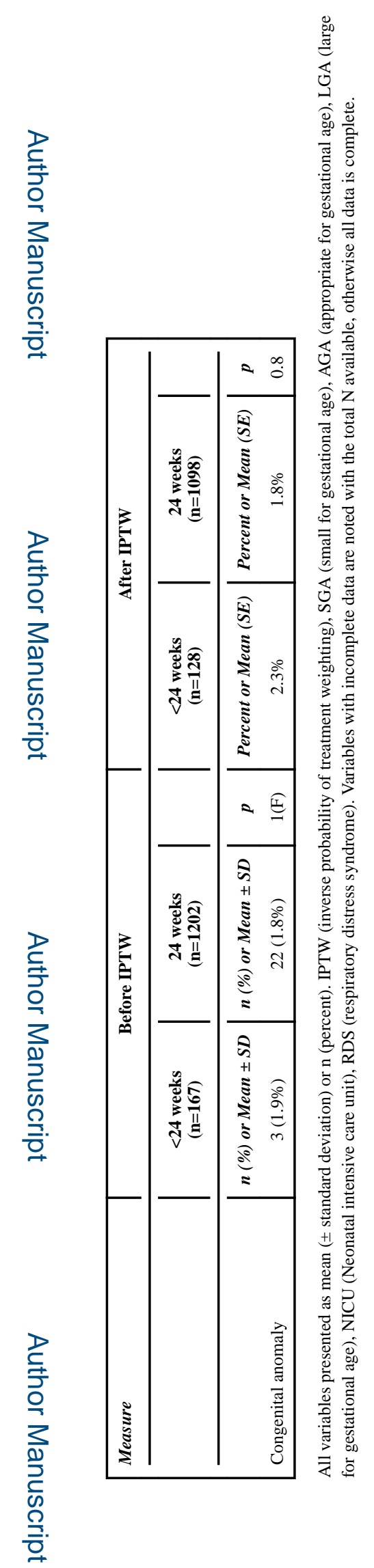

Diabetes Res Clin Pract. Author manuscript; available in PMC 2019 April 01. 


\section{Table 3}

Logistic regression analysis of outcomes associated with diagnosis of gestational diabetes mellitus prior to 24 weeks

\begin{tabular}{|l|c|c|c|c|}
\hline Measure & \multicolumn{2}{|c|}{ Before IPTW } & \multicolumn{2}{c|}{ After IPTW } \\
\hline & $\begin{array}{c}\text { OR } \\
(\mathbf{9 5 \%} \boldsymbol{C I})\end{array}$ & $\boldsymbol{p}$ & $\begin{array}{c}\text { OR } \\
(\mathbf{9 5 \%} \boldsymbol{C I})\end{array}$ & $\boldsymbol{p}$ \\
\hline Macrosomia & $\begin{array}{c}1.9 \\
(1.2,3.2)\end{array}$ & 0.01 & $\begin{array}{c}2 \\
(1,4.2)\end{array}$ & 0.0498 \\
\hline Hypertensive disorders of pregnancy & $\begin{array}{c}1.5 \\
(1,2.3)\end{array}$ & 0.04 & $\begin{array}{c}1 \\
(0.5,1.8)\end{array}$ & 0.9 \\
\hline Preterm birth & $\begin{array}{c}2.2 \\
(1.5,3.2)\end{array}$ & 0.01 & $\begin{array}{c}1.6 \\
(0.9,2.9)\end{array}$ & 0.1 \\
\hline Neonatal composite & $\begin{array}{c}1.6 \\
(1.1,2.3)\end{array}$ & 0.02 & $\begin{array}{c}1 \\
(0.6,1.7)\end{array}$ & 0.9 \\
\hline
\end{tabular}

IPTW (inverse probability of treatment weighting), OR (odds ratio), CI (confidence interval). 

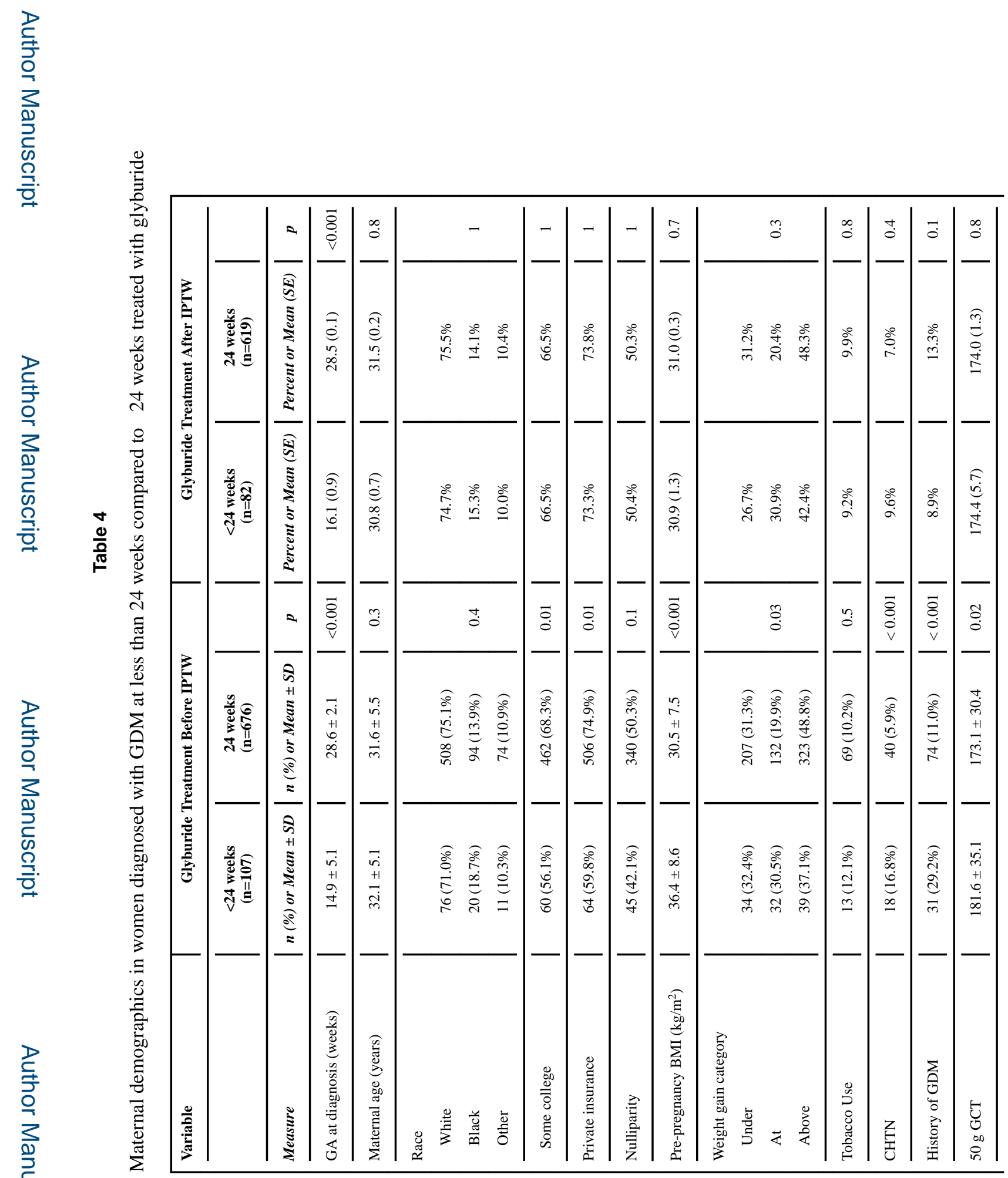

Diabetes Res Clin Pract. Author manuscript; available in PMC 2019 April 01. 


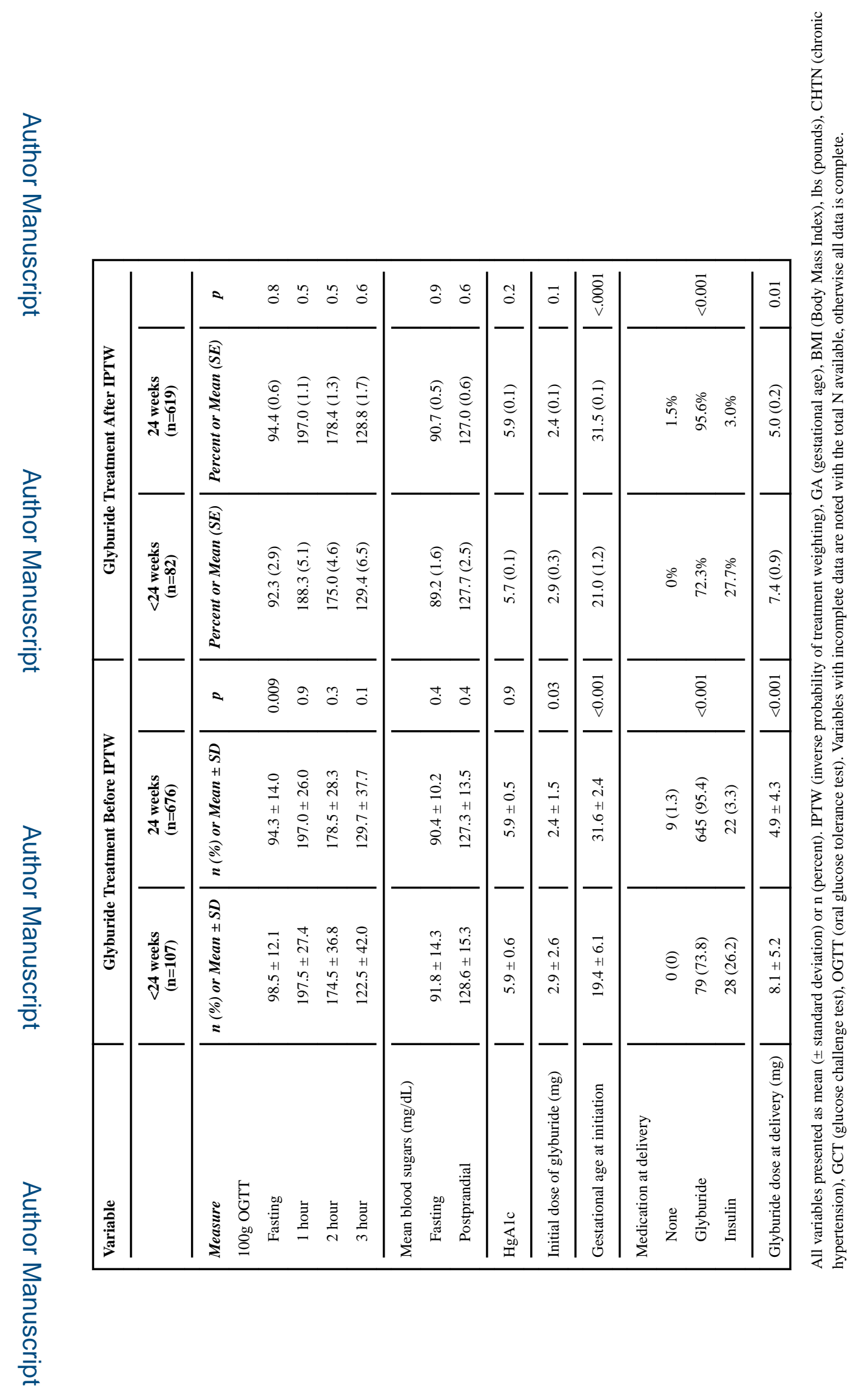

Diabetes Res Clin Pract. Author manuscript; available in PMC 2019 April 01. 


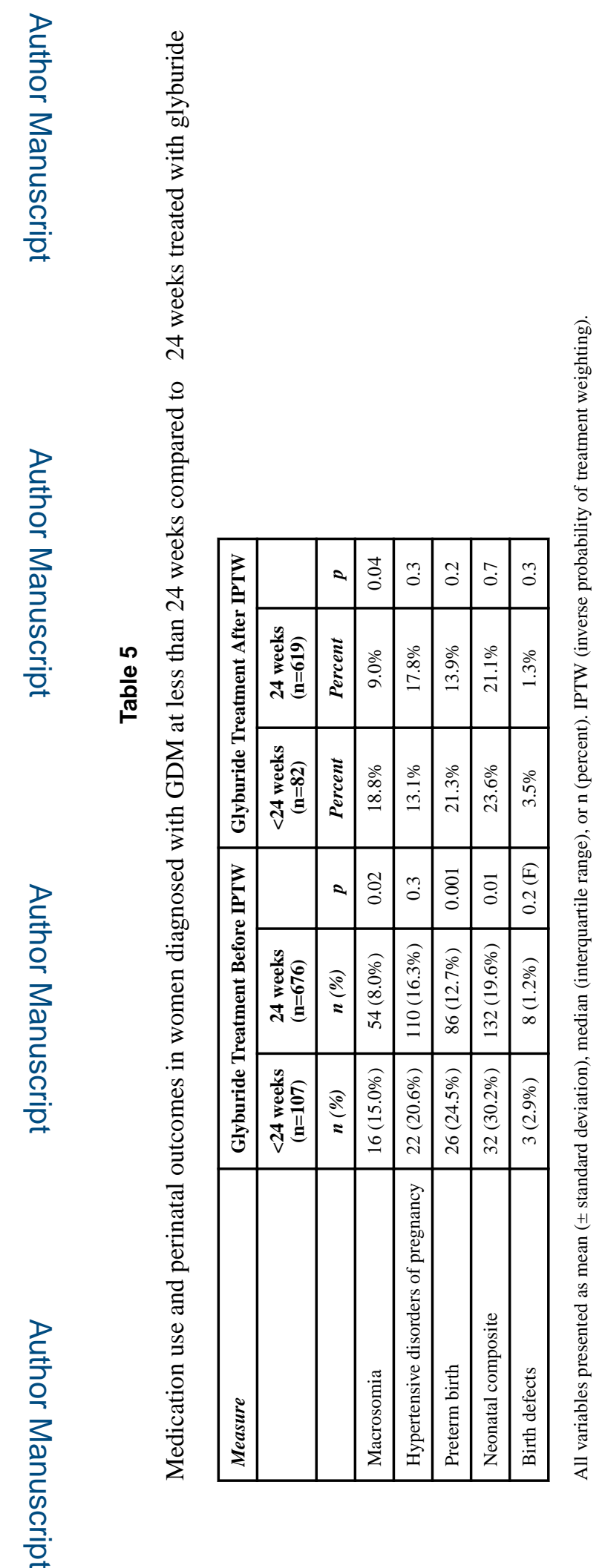

Diabetes Res Clin Pract. Author manuscript; available in PMC 2019 April 01. 


\section{Table 6}

Logistic regression analysis of outcomes associated with diagnosis of gestational diabetes mellitus prior to 24 weeks among women treated with glyburide

\begin{tabular}{|l|c|c|c|c|}
\hline Measure & \multicolumn{2}{|c|}{ Before IPTW } & \multicolumn{2}{c|}{ After IPTW } \\
\hline & $\begin{array}{c}\boldsymbol{O R} \\
\mathbf{9 5 \%} \boldsymbol{C I})\end{array}$ & $\boldsymbol{p}$ & $\begin{array}{c}\boldsymbol{O R} \\
\mathbf{9 5 \%} \boldsymbol{C I})\end{array}$ & $\boldsymbol{p}$ \\
\hline Macrosomia & $\begin{array}{c}2 \\
(1.1,3.7)\end{array}$ & 0.02 & $\begin{array}{c}2.3 \\
(1.1,5.4)\end{array}$ & 0.04 \\
\hline Hypertensive disorders of pregnancy & $\begin{array}{c}1.3 \\
(0.8,2.2)\end{array}$ & 0.3 & $\begin{array}{c}0.7 \\
(0.4,1.4)\end{array}$ & 0.3 \\
\hline Preterm birth & $\begin{array}{c}2.2 \\
(1.4,3.7)\end{array}$ & 0.02 & $\begin{array}{c}1.7 \\
(0.8,3.4)\end{array}$ & 0.1 \\
\hline Neonatal composite morbidity & $\begin{array}{c}1.8 \\
(1.1,2.8)\end{array}$ & 0.01 & $\begin{array}{c}1.2 \\
(06,2.3)\end{array}$ & 0.7 \\
\hline
\end{tabular}

IPTW (inverse probability of treatment weighting), OR (odds ratio), CI (confidence interval). IPTW (inverse probability of treatment weighting) 